\title{
Nano Bubble and Emulsion Size Distribution Measurement by Interactive Force Apparatus
}

\author{
Toyohisa FUJITA*, Takeshi KUROSE*, Josiane PONOU*, Gjergj DODBIBA*, \\ Atsushi SHIBAYAMA ${ }^{* \star}$ and Akira OTSUKI ${ }^{\star \star *}$ \\ * Department of Systems Innovation, Graduate School of Engineering, \\ The University of Tokyo, Tokyo 113-8656, Japan \\ ${ }^{* \star}$ Graduate School of International Resource Sciences, \\ Akita University, Akita 010-85026, Japan \\ *** Ecole Nationale Superieure de Geologie, Universite de Lorraine, 2 rue du Doyen Marcel Roubault, \\ TSA 70605, 54518 Vandœuvre-les-Nancy Cedex, France \\ E-mail:tfujita@sys.t.u-tokyo.ac.jp
}

\begin{abstract}
Nano bubbles are prepared by using a pressurized pump or fine ceramics in water or oil. The prepared nano bubbles are stable for more than several days. Nano emulsion (w/o type) is prepared by mixing oil, water and surfactant with homogenizer and stable more than several months. The prepared bubble and emulsion size distribution in liquid is usually measured by dynamic light scattering method. However, it is difficult to measure the particle size for smaller refractive indexes between dispersed particle and solvent by the light scattering method and small amount of particle volume. Here, the dynamical method of interactive force measurement between dispersed nano bubble and emulsion under electric field has been used to measure any kind of small particle size distribution.
\end{abstract}

Keywords : Nano bubble, Nano emulsion, Size distribution, Interactive force apparatus, Dynamic light scattering method

\section{INTRODUCTION}

There are many kinds of methods for measuring the size particles. For the particle size measurement of colloids, bubbles and emulsions, the dynamic light scattering method (DLS), laser scattering technology, resonant mass measurement method, etc. are utilized. If the particle is solid, it is possible to measure it size by mean of scanning electron microscope (SEM) and transmission electron microscope (TEM). In the size distribution measurement from nano to micro scale, the DLS is suitable. However, it is difficult to measure the particle size for smaller refractive indexes between dispersed particles and solvent by the light scattering method. Also, DLS measurement is suitable for measuring the size of only a small amount of particles. In our laboratory, the interactive force apparatus (IFA) has been studied for 15 years.

At the beginning, the interactive force apparatus is used to measure size of magnetic particles by applying magnetic field $[1,2]$. After that the dielectric particle size and coagulated particles distribution of fluorescent powders [3-5], titanium dioxide [6], silica nanoparticles [7] have been measured. In this work, the size measurements of particles in emulsion and bubbles by IFA was introduced [8]. In addition, the result SEM analysis agreed well with the results of size distribution by IFA, when Ni particles were measured [9]. Here, the fine nano bubble and emulsion size distribution measurement results by IFA were compared those of DLS analysis.

\section{EXPERIMENTAL APPARATUS AND SAMPLE}

\subsection{Measurement principle by IFA}

The arrangement of particles of different sizes in the area between flat plate and hemisphere is shown in Figure 1. The particle arrangement sometimes shows the cluster structures. The magnetic field generated by solenoid coil $[1,2]$ or electric voltage are applied between upper metallic plate and lower plate. In the measurement of bubble and emulsion size, the electric field is used. The hemisphere is employed to decide the nearest position between upper plate and lower flat plate. The interaction force between particles is the largest in the nearest position between plates.

Schematic diagram of behavior of dielectric particles dispersed in sample solution between the hemisphere and flat plate is shown

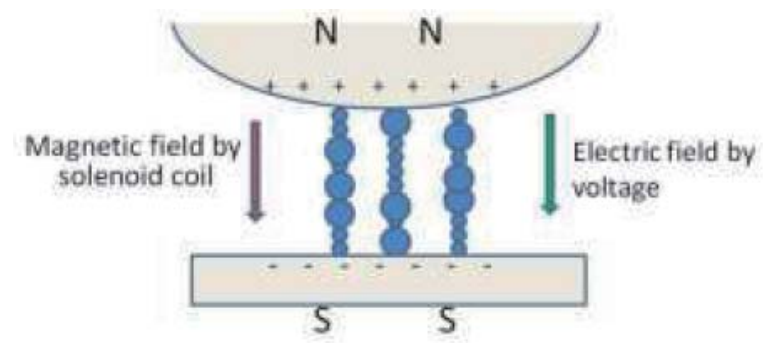

Figure 1 Arrangement of particles between flat plate and hemisphere after applying magnetic or electric field. 


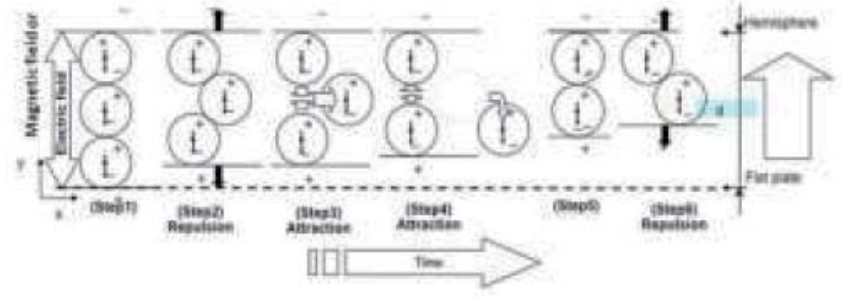

Figure 2 Schematic diagram of behavior of dielectric particles dispersed in sample solution between the hemisphere and flat plate.

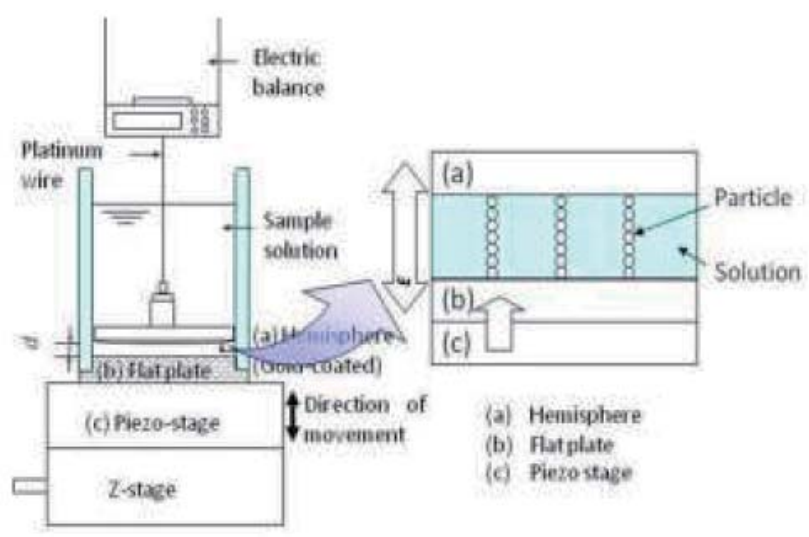

Figure 3 Schematic diagram of the main part of IFA.

in Figure 2. The minute principle is described in the former paper [9]. Schematic diagram of the main part of IFA is shown in Figure 3. Piezo stage can be moved at $5 \mathrm{~nm} / \mathrm{s}$ and moves to $1 \mu \mathrm{m}$ in this experiment.

\subsection{Sample production of bubble and emulsion}

The nano bubbles are produced by blowing pressurized nitrogen gas $(0.4 \mathrm{MPa})$ in distilled water or kerosene for 240 minutes, through fine porous ceramics. The injected bubble volume is from 0.1 to $0.2 \%$. In other words, the nano emulsion is prepared by mixing in a homogenizer with distilled water, oil (i.e. kerosene or gasoline) with nonionic or cationic surfactant [10]. It is important to note that both the water or oil samples used for the preparation of nano emulsions and bubbles are free of impurities, since it does not contain any organic molecules (such as surfactant, alcohol, etc.) or solid nano particles.

The concept of water in oil $(\mathrm{W} / \mathrm{O})$ emulsion in gasoline and nitrogen $\left(\mathrm{N}_{2}\right)$ nano bubble in distilled water is shown in Figure 4. Nano emulsion and nano bubble are stable each other for the existence of surfactant or electrical double layer. Two kinds of
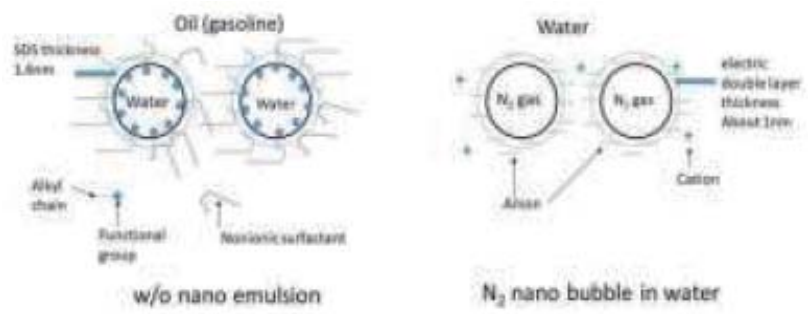

$\mathrm{N}_{2}$ nano bubble in water

Figure 4 Concept of water in oil (W/O) emulsion in gasoline and nitrogen $\left(\mathrm{N}_{2}\right)$ nano bubble in distilled water. surfactant nonionic surfactant and SDS is used to prepare w/o gasoline emulsion. The dodecyl group chain length is $1.6 \mathrm{~nm}$ [11] and nonionic surfactant are covered around the water, therefore, the water phase is coated around $2 \mathrm{~nm}$ surfactant. While the zeta potential of micro bubble is -30 to $-40 \mathrm{mV}$ and decreases from alkaline to acidic $\mathrm{pH}$ [12]. The thickness of electric double layer is about $1 \mathrm{~nm}$ in the water containing noncovalent cation and anion [13].

\subsection{Sample measurement}

In the measurement of particle size distribution, DLS and IFA methods have been used. Though DLS sometimes indicates the presence of large bubble size [8], here the results of particle size distribution measured by means of DLS are the averages of 3 different sets of measurements. On the other hand, the size measurement by means of IFA of the more than 300 particles shows that the results are stable.

\section{EXPERIMENTAL RESULTS}

\subsection{Nano bubble size distribution}

Cumulative particle size distributions of nano bubbles in distilled water measured by IFA and DLS (Otsuka electronics Co. Ltd., Japan) are shown in Figure 5. In the results by IFA under three different voltages, the higher voltage increases the particle size for the electro coagulation of bubble particles. The lower voltage of 0.1 to 0.2 is similar to the results by DLS and the mean diameter size of bubble is about $250 \mathrm{~nm}$. On the other hand, the cumulative particle size distributions of nano bubbles in kerosene measured by IFA and DLS are shown in Figure 6. The bubble size distribution in kerosene is almost same by IFA at from 0.1 to $0.2 \mathrm{~V}$. However, the bubble size in kerosene by DLS becomes larger. The reflection index difference between $\mathrm{N}_{2}$ and oil becomes smaller comparing water; the measured value by DLS might be changed.

\subsection{Nano emulsion size distribution}

The result of cumulative water particle size distribution in light oil of w/o emulsion by IFA and DLS is shown in Figure 7. The $50 \%$ size mean diameter is approximately $7 \mathrm{~nm}$ in both DLS and IFA. The size distribution by IFA is broader than DLS. However, the water particle size distribution by DLS agreed well with the IFA.

The reflection index of water is 1.33 in $590 \mathrm{~nm}$ and the oil like kerosene is usually 1.45 to 1.48 [13]. As the difference of reflection

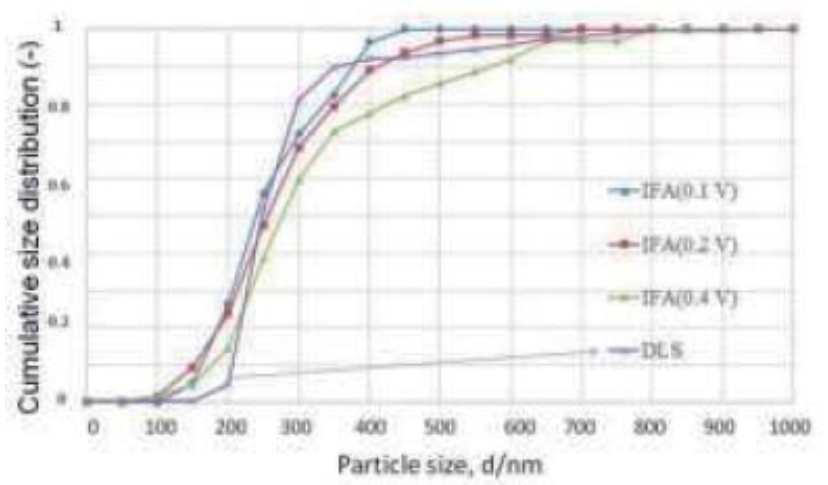

Figure 5 Cumulative particle size distribution of nano bubbles in distilled water measured by IFA under the 3 different applied voltages, and measured by DLS 


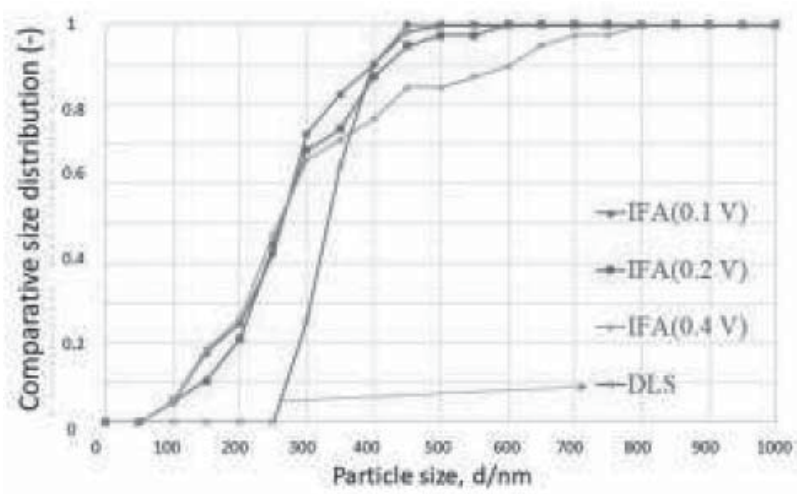

Figure 6 Cumulative particle size distribution of nano bubbles in kerosene measured by IFA under the 3 different applied voltages, and measured by DLS

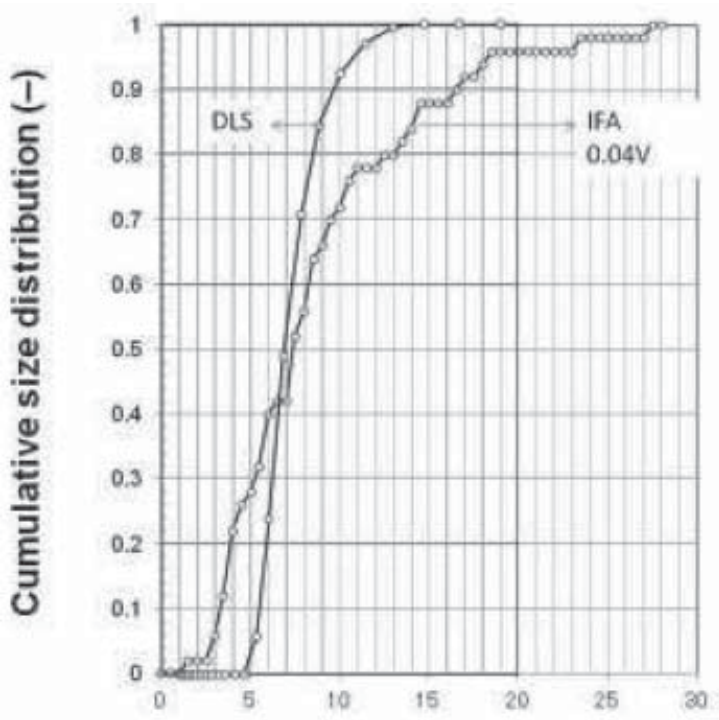

Particle size, $d / \mathrm{nm}$

Figure 7 Cumulative particle size distribution of water in kerosene (light oil) of w/o emulsion by IFA and DLS.

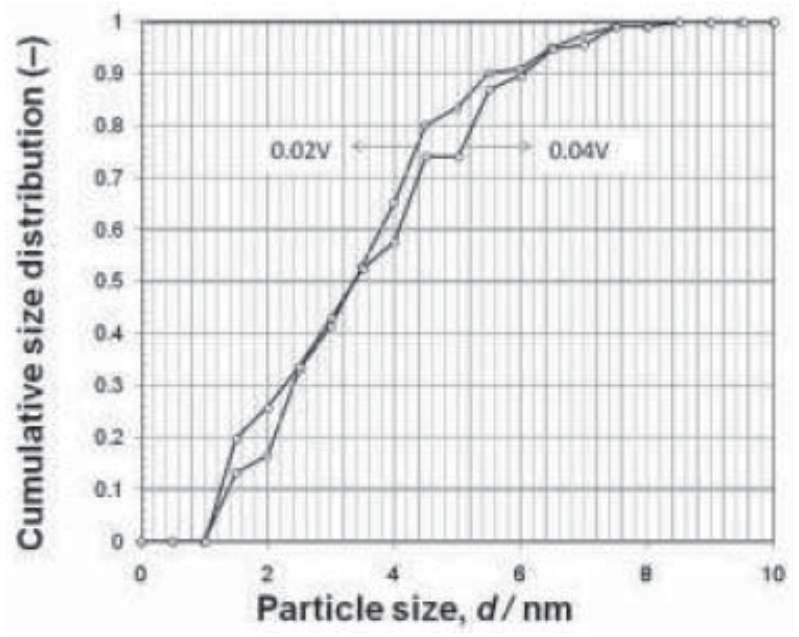

Figure 8 Cumulative water particle size distribution of gasoline in w/ o emulsion measured by IFA shown in Figure 2 at different voltages (i.e. 0.02 and $0.04 \mathrm{~V}$ ) between plates. index is large, DLS to measure the particle size in emulsion is possible. However, the reflection index of gasoline is 1.40 and the reflection index difference is small. It is difficult to measure the water particle size by DLS. The physical method by IFA is possible to measure the water particle size distribution in emulsion. The results by applying 0.02 and 0.04 voltages between plates are shown in Figure 8 . The $50 \%$ mean diameter of water particles in gasoline of w/o emulsion is very fine, i.e. about $3.5 \mathrm{~nm}$ in both fields. The w/o emulsion containing $20 \%$ of water in gasoline has been stable for one year [14].

\section{CONCLUSIIONS}

The nano to micro emulsion particle size is usually measured by light scattering method (DLS). Here, the interactive force apparatus (IFA) by physical method could be measured the size distribution of nano bubbles and nano emulsion. The particle size distribution of nano bubbles and nano emulsion measured by IFA agreed well with the ones by DLS. While, the bubble size in kerosene by DLS becomes larger. DLS affects the reflection index difference between dispersed particle and solvent, while IFA can measured the size of various kinds of bubbles and emulsions. The IFA can be measure the size of any kind of fine particles in air, or any kind of liquid or solvent giving more stable results, when comparing with DLS.

\section{References}

[1] Miyazaki T., Shibayama A., Sato T., Fujita T., "Measurement of Interaction Force between Small Distances Sandwiched with Magnetic Fluid under Magnetic Field", J. Magnetism and Magnetic Materials, 252, 256-258 (2002).

[2] Miyazaki T., Shibayama A., Fujita T., "Measurement of Surface force of fine particles at a short distance in a magnetic fluid under an electric and magnetic field", J. of the Magnetics Soc. Japan, 26, 3, 153-156 (2002), (in Japanese).

[3] Otsuki A., Dodbiba G., Fujita T., "Effect of particle size distribution on formation of linear configuration of dielectric fine particles undre the electric field", J. Physics: Conference Series 147, 012003, 1-4 (2009).

[4] Otsuki A., Dodbiba G., Shibayama A., Sadaki J., Mei G., Fujita T., "Separation of Rare Earth Fluorescent Powders by Two-Liquid Flotation using Organic Solvent", J. J. Applied Physics, 47, No.6, 5093-5099 (2008).

[5] Otsuki A., Sadaki J., Yamaguchi K., Shibayama A., Fujita T., "Observation of Aggregate Structure of Green and Blue Fluorescent Powders Suspended in Heptane by Interactive Force Measurement", Int. J. Soc. Mater. Eng. Resour., 13, No.2, 86-91 (2006).

[6] Otsuki A., Dodbiba G., Fujita T., “Measurements of size distribution of titanium dioxide fine particles in a highly concentrated non-aqueous suspension by using particle self- assembly under an electric field", Advanced Powder Technology, 23, 517-522 (2012).

[7] Otsuki A., Dodbiba G., Fujita T., "Measurements of particle size distribution of silica nano particles by interactive force apparatus under an electric field", Advanced Powder Technology, 21, 419-423 (2010)

[8] Fujita T., Dodbiba G., Ponou J., Shibayama A., Otsuki A., "Fine particle liquid and bubble size distribution measurement in liquids”, IMPC 2016; XXVIII International Mineral Processing Congress Proceedings-ISBN:978-1-926872-29-2, 1-9 (2016). 
[9] Fujita T., Dodbiba G., Okaya K., Matsuo S., Wang L. P., Onda K. and Otsuki A., "Nano to micro size distribution measurement in the fluid by interactive force apparatus for fine particle processing", J. Nanoscience and Nanotechnology, 13, 8184-8189 (2013).

[10] Fujita T., Dodbiba G., Ponou J., Udaeta M.C., "Reduction of $\mathrm{PM}, \mathrm{NOX}, \mathrm{CO}$ in the emitted gas and increase of combustion efficiency using stable emulsion fuel", ICATSD2016 -ISGT 2016, 1-10, Ho-chi Minh city, Vietnam,1339-1346 (2016).

[11] Horiguchi H., "New surfactant", Sankyo Shuppan Co., Ltd.,
Tokyo, P.52 (1975).

[12] Edited by Ishii T. "Concepts in Basic Bubble and Foam Engineering”, Technosystem Co., Ltd, p.466 (2005).

[13] Translation by Kitahara F. and Aoki K., "Introduction to Colloid and Surface Chemistry”, HIrokawa-books, Co., Ltd., Tokyo, p.148 (1983).

[14] Handbook of Chemistry and Physics, $89^{\text {th }}$ edition, CRC press, New York, (2008).

[15] Japanese Unexamined Patent Application Publication No.2012-72349. 